\title{
Early decline spectra of nova SMC 2001 and nova LMC 2002
}

\author{
E. Mason ${ }^{1}$, M. Della Valle ${ }^{2}$, R. Gilmozzi ${ }^{1}$, G. Lo Curto ${ }^{1}$, and R. E. Williams ${ }^{3}$ \\ 1 ESO, Alonso de Cordova 3107, Casillas 19001, Vitacura, Santiago, Chile \\ e-mail: emason@eso.org \\ 2 Osservatorio Astronomico di Arcetri, Largo E. Fermi 5, Firenze, Italy \\ 3 Space Telescope Science Institute, 3700 San Martin Drive, Baltimore, MD, USA
}

Received 25 May 2004 / Accepted 22 November 2004

\begin{abstract}
We report results on the spectroscopic follow-up of Nova SMC 2001 and Nova LMC 2002 carried out at La Silla. The analysis of the spectroscopic evolution shows that these objects belong to the Fe II class, according to the Cerro Tololo scheme. From the line fluxes and the expansion velocities, we have derived an approximate mass for the ejected shells of $2 \div 3 \times 10^{-4} M_{\odot}$. The filling factor measurements $\left(\varepsilon \sim 10^{-4} \div 10^{-1}\right)$ suggest a clumpy structure for the ejecta.
\end{abstract}

Key words. stars: novae, cataclysmic variables - stars: individual: Nova LMC 2002, Nova SMC 2001

\section{Introduction}

Spectroscopic observations of extra-galactic novae are relatively rare. In this paper we report the spectroscopic evolution of two novae that recently occurred in the Magellanic Clouds: Nova SMC 2001 and Nova LMC 2002. These data are doubly interesting: they provide important information on the nova event itself and help to characterize the properties of nova populations in galaxies that have different metallicity content, luminosity class and Hubble type. The existence of systematic differences among extra-galactic novae has been recognized on the basis of the observed nova counts (Duerbeck 1990), the distribution of the photometric speed classes (Della Valle et al. 1992), the frequency of occurrence of nova events in different Hubble type galaxies (Della Valle et al. 1994; Williams \& Shafter 2004) and the spectroscopic evolution (Della Valle \& Livio 1998).

Nova SMC 2001 was discovered by Liller (2001) on Oct. 21.09 UT at a magnitude $V=12.21$. The first spectra were taken on Oct. 26.15 UT and 30.25 UT by Bosch et al. (2001) and Della Valle et al. (2001). They reported the detection of strong Balmer and low ionization emission lines (e.g., FeII, OI, and $\mathrm{NaI}$ ), flanked by P-Cyg profiles which indicated an expansion velocity of $950 \mathrm{~km} \mathrm{~s}^{-1}$ from the P-Cyg absorption of the FeII and NaI lines, while velocities of 1400 and $1800 \mathrm{~km} \mathrm{~s}^{-1}$ were derived from the P-Cyg absorption of the Balmer lines $\mathrm{H} \alpha$ and $\mathrm{H} \beta$, respectively. Jensen et al. (2001) reported the detection of CII in a spectrum obtained on Nov. 2.12 UT and pointed out that the nova strongly resembles Nova LMC 1988 No. 1 two weeks after maximum. This may suggests that nova SMC 2001 reached maximum light around October 15.

Nova LMC 2002 was discovered by Liller (2002) on March 3 when the object was close to maximum brightness.

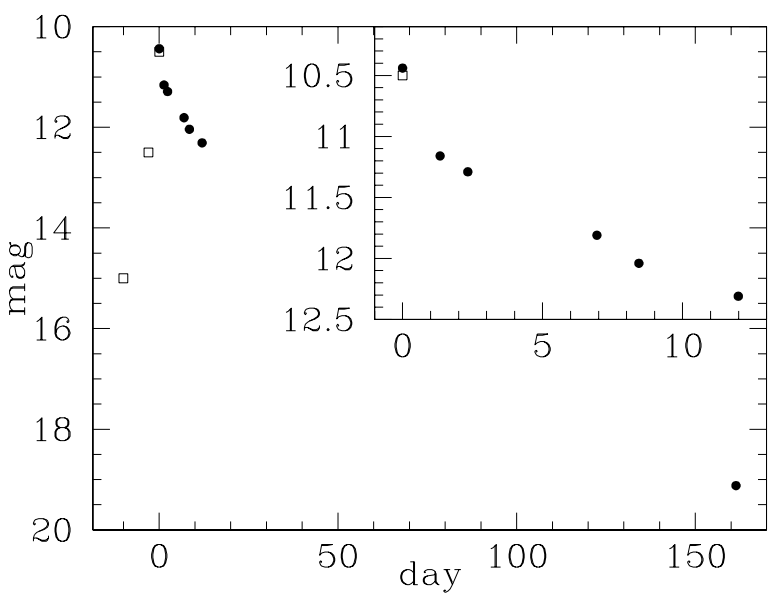

Fig. 1. Nova LMC 2002 light curve. Day 0 is the day of the observed maximum. The upper right box is a zoomed-in view of the light curve. Squares in the rising part of the curve are photographic magnitudes, filled circles are $V$ band magnitudes.

The nova was also detected in two pre-discovery images (Liller 2002) obtained on February 21 and $27\left(m_{\mathrm{pg}} \sim 15\right.$ and $m_{\mathrm{pg}}=12.5$, respectively). In the first low resolution spectrum, taken on March 4, Liller (2002) reports the presence of strong $\mathrm{H} \alpha$ and $\mathrm{H} \beta$ emission. After Liller's discovery there are only few photometric observations by Kilmartin \& Gilmore (2002) and Gilmore (2002). We plot in Fig. 1 the visual photometry in the IAU Circulars (No. 7841, 7847 and 7853) and the $V$ magnitudes estimated from our spectra (see Sect. 3 and Table 4).

We present our spectroscopic observations in Sect. 2. The data and their analysis are reported in Sect. 3. The summary and the conclusion follow in Sect. 4. 
Table 1. The log of observations of Nova SMC 2001.

\begin{tabular}{|c|c|c|c|c|c|c|}
\hline Obs. date & UT start & Exp. time (s) & Telescope+Instrument & Mode & $\lambda$ range & $\AA ̊$ /pix \\
\hline $01 / 11 / 2001$ & $01: 12$ & 1800 & 1.5+FEROS & (echelle) & $3900-9200$ & 1.0 \\
\hline \multirow[t]{8}{*}{$06 / 12 / 2001$} & 01:20 & 3722 & NTT+EMMI & DIMD2, grating\#3 & $3300-3750$ & 0.45 \\
\hline & $02: 27$ & 2522 & & & $3500-4000$ & 0.45 \\
\hline & $03: 15$ & 2522 & & & $4000-4500$ & 0.45 \\
\hline & 04:02 & 2522 & & & $4500-5000$ & 0.45 \\
\hline & 01:20 & 3600 & & DIMD1, grating\#7 & $4800-6200$ & 0.67 \\
\hline & $02: 27$ & 2522 & & & $6150-7500$ & 0.65 \\
\hline & $03: 16$ & 2400 & & & $7450-8750$ & 0.65 \\
\hline & 04:02 & 2400 & & & $8750-10050$ & 0.64 \\
\hline \multirow[t]{3}{*}{$17 / 02 / 2002$} & 02:01 & 300 & NTT+EMMI & RILD, grism\#1 & $3600-10200$ & 5.8 \\
\hline & 01:03 & 1200 & & RILD, grism\#5 & $4000-6650$ & 1.3 \\
\hline & $01: 27$ & 1800 & & RILD, grism\#6 & $6000-8350$ & 1.2 \\
\hline \multirow[t]{3}{*}{$4 / 03 / 2002$} & $01: 27$ & 600 & NTT+EMMI & RILD, grism\#1 & $3600-10200$ & 5.8 \\
\hline & $00: 16$ & 1550 & & RILD, grism\#5 & $4000-6650$ & 1.3 \\
\hline & 00:46 & 2265 & & RILD, grism\#6 & $6000-8350$ & 1.2 \\
\hline
\end{tabular}

Table 2. The log of observations of Nova LMC 2002.

\begin{tabular}{ccccccc}
\hline \hline Obs. date & UT start & Exp. time $(\mathrm{s})$ & Telescope+Instrument & Mode & $\lambda$ range $(\AA)$ & $\AA /$ pix \\
\hline $09 / 03 / 2002$ & $23: 55,00: 00,00: 11$ & $40+60 \times 2$ & $3.6+$ EFOSC & grism\#9 & $4700-6750$ & 2.0 \\
& $23: 59,00: 08,00: 19$ & $20+35 \times 2$ & & grism\#12 & $6000-10300$ & 4.2 \\
& $23: 56,00: 02,00: 12$ & $40+100 \times 2$ & & grism\#14 & $3200-5100$ & 2.0 \\
& $23: 57,00: 04,00: 14$ & $40+200 \times 2$ & & grism\#15 & $6900-8750$ & 1.9 \\
$14 / 03 / 2002$ & $01: 14,01: 24$ & $60+120$ & $3.6+$ EFOSC & grism\#9 & $4700-6750$ & 2.0 \\
& $01: 22,01: 37$ & $35+70$ & & grism\#12 & $6000-10300$ & 4.2 \\
& $01: 15,01: 26$ & $100+200$ & & grism\#14 & $3200-5100$ & 2.0 \\
& $01: 26,01: 30$ & $200+400$ & & grism\#15 & $6900-8750$ & 1.9 \\
$11 / 08 / 2002$ & $10: 15$ & 900 & NTT+EMMI & RILD, grism\#3 & $3850-9100$ & 2.8 \\
\hline
\end{tabular}

\section{Observations}

Spectroscopic observations for Nova SMC 2001 and Nova LMC 2002 were taken in service mode during the target of opportunity campaign at the $1.5 \mathrm{~m}+$ FEROS, $3.6 \mathrm{~m}+\mathrm{EFOSC}$, and NTT+EMMI, at La Silla Observatory. Details of the $\log$ of observations are in Tables 1 and 2 for Nova SMC 2001 and Nova LMC 2002, respectively. Spectrophotometric standard stars were taken every night except November 1 . Screen flats, bias, and wavelength calibration frames were secured on the day following each observing run. The spectra were reduced using standard IRAF routines.

\section{Spectral analysis}

\subsection{The observed evolution}

We identified the emission lines and measured the corresponding fluxes in order to classify the novae according to the Cerro Tololo system (Williams et al. 1991, 1994), and to provide input parameters for the computation of the elemental abundances (e.g., Shore et al. 2003). The observed spectroscopic evolution of each nova is described below and summarized in Table 3.
Table 3. The observed spectroscopic evolution of Nova SMC 2001 and Nova LMC 2002 according to the Cerro Tololo classification. For Nova SMC 2001 we write in brackets the spectroscopic phase reported in the IAUC No. 7744.

\begin{tabular}{ll}
\hline \hline Object & Spec. ev. \\
\hline Nova SMC 2001 & $\left(P_{\mathrm{fe}}^{o}\right), P_{n a / n}^{o}, P_{n}^{o}, A_{o}$ \\
Nova LMC 2002 & $P_{\mathrm{fe}}, A_{o}$ \\
\hline
\end{tabular}

For both novae we estimated the $V$ band magnitude at each epoch (see Table 4) by convolving the observed spectra with the $V$-Johnson filter. The synthetic magnitude of Nova SMC 2001 obtained on March 4 (2002) may indicate that the nova was undergoing a re-brightening of at least one magnitude, thus suggesting the formation of dust within the ejecta similar to FH Ser, NQ Vul, and Nova Aql 1982 (Hack et al. 1993). However, since the sky conditions during the observation were not reported, we cannot rule out that the sky was not photometric, and that the re-brightening is an artifact of the calibration. The line flux measurements for Nova SMC 2001 and Nova LMC 2002 are given in Tables 5 and 6, respectively. 
Table 4. The instrumental magnitudes derived by convolution of the observed spectra with the standard $V$-Johnson filter.

\begin{tabular}{|c|c|}
\hline Date & $\bar{V}$ \\
\hline \multicolumn{2}{|c|}{ Nova SMC 2001} \\
\hline $06 / 12 / 01$ & 15.3 \\
\hline $17 / 02 / 02$ & 16.4 \\
\hline 04/03/02 & 15.4 \\
\hline \multicolumn{2}{|c|}{ Nova LMC 2002} \\
\hline $09 / 03 / 02$ & 11.8 \\
\hline $14 / 03 / 02$ & 12.3 \\
\hline $11 / 08 / 02$ & 19.1 \\
\hline
\end{tabular}

\section{Nova SMC 2001}

The spectroscopic follow-up of Nova SMC 2001 covers days $11,46,119$, and 134 since the observed maximum (Fig. 2).

The spectrum taken in November 2001 (11 days past maximum) is dominated by the Balmer lines and OI 18446 . We also observed permitted transitions from both low (FeII, NaI, and $\mathrm{MgII}$ ) and moderate excitation lines (HeI, CII, and NII). Forbidden transitions from [OI] and [NII] have been detected, too. The Balmer lines $\mathrm{H} \beta, \mathrm{H} \gamma$ and $\mathrm{H} \delta$ show double P-Cyg absorptions whose minima indicate average expansion velocities of -1300 and $-1000 \mathrm{~km} \mathrm{~s}^{-1}$. The FeII (42) emission lines show just a single P-Cyg absorption, indicating an average expansion velocity of $\sim-900 \mathrm{~km} \mathrm{~s}^{-1}$.

In December 2001 (46 days past maximum) Nova SMC 2001 exhibited the [OIII] lines $\lambda \lambda 4959,5007$ which characterizes the "nebular phase" of novae (Payne-Gaposchkin 1957). The line profiles are clearly all saddle shaped with the red component $(R)$ stronger than the blue one $(B)$, with the exception of the $\mathrm{H} \beta$ line for which $B>R$ (see Fig. 3). The separation between the red and the blue component is $\sim 900 \div 1000 \mathrm{~km} \mathrm{~s}^{-1}$, whereas, the $F W H M$ of the emission lines is $\sim 1400 \div 1900 \mathrm{~km} \mathrm{~s}^{-1}$. Saddle shaped profiles are suggestive of an expanding equatorial shell or ejection of polar caps (Payne-Gaposchkin 1957).

The spectra taken on February and March 2002 (119 and 164 days past maximum, respectively) are quite similar and are dominated by strong auroral and nebular emission lines such as [OIII] $\lambda 5007$ (as strong as the $\mathrm{H} \alpha$ emission), and [OIII] $\lambda 4363$. All the permitted lines have considerably weakened, while the " 4640 " blend is now clearly resolved into

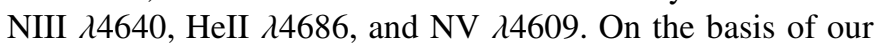
spectroscopic observations we classify the spectra obtained in November and December 2001 as $P_{n a / n}^{o}$ and $P_{n}^{o}$, respectively, and those taken in February and March 2002 as $A_{o}$, according to the Cerro Tololo scheme.

\section{Nova LMC 2002}

We obtained spectra of nova LMC 2002 6, 11 and 161 days after discovery (see Fig. 4 and Table 6). From the light curve in Fig. 1 we derive $t_{2}=12$ days, which makes Nova LMC 2002 a borderline object between the fast and slow nova classes as defined by Della Valle \& Livio (1998).
The early spectra ( 6 and 11 days past maximum) are dominated by Balmer and low ionization emission lines such as FeII, OI, CaII, MgII and NaI D. Weak emission lines from CI, CII and NI are detected red-ward of $\sim 7000 \AA$. We also observe the [OI] $\lambda 5577$ and $\lambda \lambda 6300,6364$ emission lines. These spectra are quite similar to those of Nova LMC 1988 No. 1 three days after maximum, and Nova Scuti 1989 fifteen days after maximum. We classify our spectra as $P_{\text {fe }}$ according to the Cerro Tololo system.

The spectrum taken in August 2002 (161 days past maximum) is dominated by strong auroral and nebular lines from [OIII] and [NII]. All low ionization emission lines have completely disappeared, and the only permitted transitions are due to NII, HeII, CII, NIII, CIV, OI and OII. At this stage the spectrum corresponds to the $A_{o}$ phase in the Cerro Tololo system. This fact, coupled with an expansion velocity of $2150 \mathrm{~km} \mathrm{~s}^{-1}$, as measured from the $\langle H W Z I\rangle$ of the Balmer lines near maximum, indicates that Nova LMC 2002 belongs to the FeII spectroscopic class (indeed HWZIs smaller than $2500 \mathrm{~km} \mathrm{~s}^{-1}$ are typical of FeII type novae, see Williams 1992).

\subsection{Reddening, flux measurements and the neutral oxygen mass}

The emission line fluxes of dereddened spectra at each epoch are reported in Tables 5 and 6 for Nova SMC 2001 and Nova LMC 2002, respectively. The correction for extinction was applied assuming $R=3.1$, and $E(B-V)=0.09( \pm 0.02)$ and $0.13( \pm 0.05)$ for Nova SMC 2001 and Nova LMC 2002, respectively (see also Sect. 3.4). The values for $E(B-V)$ were computed by averaging the extinction estimates in the literature (Dutra et al. 2001; Oestreicher \& Schmidt-Kaler 1996; Oestreicher et al. 1995; Massey et al. 1995; Schwering \& Israel 1991; Bessel 1991; and Capaccioli et al. 1990). Extinction values derived from extinction maps of the Magellanic Clouds were weighted twice in our computation.

The calculated correction, however, does not take into account the internal extinction of each nova, which can be higher due to dust formation. This can be checked by using the intensity ratio of optically thin lines which have the same upper level. We could use the $\mathrm{H}(8) / \mathrm{HP}(8)$ line ratio measured in the December spectrum of Nova SMC 2001. We derived an extinction correction of $E(B-V)=0.26 \pm 0.20$ (cf. Vanlandingham et al. 1999), which is indeed larger than the above value of $0.09 \pm 0.02$, although still consistent within the errors.

Temperature, $T_{\mathrm{e}}$, and mass of the neutral oxygen in the ejecta have been computed following Williams (1994). In case of relatively high densities (as in novae ejecta) the [OI] lines $\lambda 5577$ and $\lambda 6300$ are easily detectable and can be used as a diagnostic of the electron temperature. After substituting in the equation for the line flux ratio the Einstein coefficients, the excitation potential and the statistical weight of the energy levels (see Williams 1994, and references therein) we get:

$T_{\mathrm{e}}=\frac{11200}{\log \left(\frac{43 \tau}{\left(1-\mathrm{e}^{-\tau}\right)} \times \frac{F_{\lambda 6300}}{F_{\lambda 5577}}\right)}$ 
Table 5. Nova SMC 2001 emission line fluxes. The absolute fluxes for November 2001 have been derived from the relative fluxes after assuming $t 2 \sim 20$ days. Flux measurements are affected by errors of the order of $\sim 50 \%$ (Col. 2) and $\sim 30 \%$ (Cols. 3-5).

\begin{tabular}{|c|c|c|c|c|}
\hline Line & 01-11-2001 & 06-12-2001 & $17 / 18-02-2002$ & 4/5-03-2002 \\
\hline H18(4) 3692 & - & $7.54 \mathrm{E}-15$ & - & - \\
\hline H17(3) 3697 & - & $1.58 \mathrm{E}-14$ & & \\
\hline H13(3) 3734 & - & $3.96 \mathrm{E}-14$ & - & - \\
\hline H12(2) 3750 & - & $7.63 \mathrm{E}-14$ & - & - \\
\hline H11(2) 3771 & - & $9.25 \mathrm{E}-14$ & - & - \\
\hline H10(2) 3798 & - & $6.88 \mathrm{E}-14$ & - & - \\
\hline $\mathrm{H} \eta 3835$ & - & $1.16 \mathrm{E}-13$ & - & - \\
\hline $\mathrm{H} \zeta 3889$ & - & $1.44 \mathrm{E}-13$ & - & - \\
\hline Н $\epsilon 3970$ & $\begin{array}{l}1.58 \mathrm{E}-12 \\
\text { CaII }(1)\end{array}$ & $+\quad 1.54 \mathrm{E}-13$ & $6.37 \mathrm{E}-14$ & - \\
\hline HeI(18) 4026 & - & - & $1.10 \mathrm{E}-14$ & - \\
\hline [SII](1) 4068 & - & - & $3.05 \mathrm{E}-14$ & $3.21 \mathrm{E}-14$ \\
\hline $\mathrm{H} \delta 4102$ & $4.83 \mathrm{E}-12$ & $3.19 \mathrm{E}-13$ & $1.44 \mathrm{E}-13$ & $1.35 \mathrm{E}-13$ \\
\hline FeII(28) 4179 & $3.89 \mathrm{E}-12$ & - & - & - \\
\hline HeII(3) 4200 & - & - & $1.61 \mathrm{E}-14$ & $1.34 \mathrm{E}-14$ \\
\hline FeII(27) 4233 & $1.02 \mathrm{E}-12$ & $1.16 \mathrm{E}-14$ & - & - \\
\hline CII(6) 4267 & $7.37 \mathrm{E}-13$ & $5.10 \mathrm{E}-14$ & $2.03 \mathrm{E}-14$ & - \\
\hline $\mathrm{H} \gamma 4341$ & $4.71 \mathrm{E}-12$ & $5.145 \mathrm{E}-13$ & $1.29 \mathrm{E}-13$ & $1.218 \mathrm{E}-13$ \\
\hline [OIII](2) 4363 & - & - & $4.77 \mathrm{E}-13$ & $3.91 \mathrm{E}-13$ \\
\hline FeII(27) 4417 & $7.78 \mathrm{E}-13$ & $2.47 \mathrm{E}-14$ & - & - \\
\hline$[\mathrm{FeII}](7) 4452$ & $1.64 \mathrm{E}-13$ & $2.33 \mathrm{E}-14$ & - & - \\
\hline $\operatorname{HeI}(14) 4471$ & - & - & $1.47 \mathrm{E}-14$ & \\
\hline$[\mathrm{FeII}](7) 4475$ & $2.26 \mathrm{E}-13$ & $3.62 \mathrm{E}-14$ & - & - \\
\hline $\operatorname{MgII}(4) 4481$ & $1.64 \mathrm{E}-12$ & - & - & - \\
\hline FeII(37) 4515/20 & $1.64 \mathrm{E}-13$ & - & - & - \\
\hline NIII(3) 4511/15/24 & - & - & $2.77 \mathrm{E}-14$ & $2.65 \mathrm{E}-14$ \\
\hline FeII(38) 4523/40 & $4.71 \mathrm{E}-13$ & - & - & - \\
\hline FeII(38) $4550+(37) 4556$ & $2.05 \mathrm{E}-13$ & - & - & - \\
\hline FeII(38) 4584 & $3.48 \mathrm{E}-13$ & - & - & - \\
\hline NII(5) 4607/14 & - & - & $3.03 \mathrm{E}-14$ & $3.01 \mathrm{E}-14$ \\
\hline NIII(2) $4641 / 2+$ CIII(1) 4650 & - & $9.57 \mathrm{E}-13$ & $1.40 \mathrm{E}-13$ & $1.36 \mathrm{E}-13$ \\
\hline OII(1) 4651/61 & $1.47 \mathrm{E}-12$ & - & - & - \\
\hline HeII(1) 4686 & - & - & $5.13 \mathrm{E}-13$ & $4.81 \mathrm{E}-14$ \\
\hline $\operatorname{NII}(20) 4803 / 10$ & - & - & $7.43 \mathrm{E}-15$ & $5.43 \mathrm{E}-15$ \\
\hline $\mathrm{H} \beta 4861$ & $1.29 \mathrm{E}-11$ & $8.13 \mathrm{E}-13$ & $2.25 \mathrm{E}-13$ & $2.37 \mathrm{E}-13$ \\
\hline HeI(48) 4922 & - & $1.17 \mathrm{E}-13$ & $1.044 \mathrm{E}-13$ & - \\
\hline FeII(42) 4924 & $3.89 \mathrm{E}-12$ & - & - & - \\
\hline [OIII]1 4959 & - & $5.82 \mathrm{E}-14$ & $2.70 \mathrm{E}-13$ & $3.67 \mathrm{E}-13$ \\
\hline [OIII]1 5007 & - & $4.05 \mathrm{E}-13$ & $7.78 \mathrm{E}-13$ & $1.06 \mathrm{E}-12$ \\
\hline HeI(4) 5016 & - & - & $2.00 \mathrm{E}-14 \mathrm{~b}$ & $1.8 \mathrm{E}-14 \mathrm{~b}$ \\
\hline FeII(42) 5018 & $4.91 \mathrm{E}-12$ & - & - & - \\
\hline FeII(42) 5169 & $4.10 \mathrm{E}-12$ & - & - & - \\
\hline NII(66) 5176/80 & - & $4.19 \mathrm{E}-14$ & $1.33 \mathrm{E}-14$ & $9.80 \mathrm{E}-15$ \\
\hline FeII(49) 5198 & $1.13 \mathrm{E}-12$ & - & - & - \\
\hline FeII(49) 5235 & $7.57 \mathrm{E}-13$ & - & - & - \\
\hline FeII(49) 5276 & $1.27 \mathrm{E}-12$ & $2.96 \mathrm{E}-14$ & - & - \\
\hline FeII(49) 5317 & $2.25 \mathrm{E}-12$ & - & - & - \\
\hline FeII(48) 5363 & $1.04 \mathrm{E}-12$ & - & $1.369 \mathrm{E}-15$ & $1.569 \mathrm{E}-15$ \\
\hline HeII(2) 5412 & - & - & $3.53 \mathrm{E}-15$ & $3.54 \mathrm{E}-15$ \\
\hline NII(29) 5463/80/96 & - & $2.23 \mathrm{E}-14$ & $4.10 \mathrm{E}-15$ & $3.56 \mathrm{E}-15$ \\
\hline FeII(55) 5535 & $9.62 \mathrm{E}-13$ & - & - & - \\
\hline [OI](3) 5577 & $3.89 \mathrm{E}-12$ & $3.55 \mathrm{E}-14$ & $2.54 \mathrm{E}-15$ & $2.81 \mathrm{E}-15$ \\
\hline NII(3) 5666/80 & $1.02 \mathrm{E}-11$ & $1.39 \mathrm{E}-13$ & $2.62 \mathrm{E}-14$ & $2.66 \mathrm{E}-14$ \\
\hline
\end{tabular}


Table 5. continued.

\begin{tabular}{|c|c|c|c|c|}
\hline Line & 01-11-2001 & 06-12-2001 & 17/18-02-2002 & $4 / 5-03-2002$ \\
\hline$[\mathrm{NII}](3) 5755$ & $1.15 \mathrm{E}-11$ & $4.04 \mathrm{E}-13$ & $1.53 \mathrm{E}-13$ & $2.05 \mathrm{E}-13$ \\
\hline CIV(1) 5802/12 & - & $1.16 \mathrm{E}-13$ & $5.36 \mathrm{E}-15$ & $4.99 \mathrm{E}-15$ \\
\hline HeI(11) 5876 & - & $1.25 \mathrm{E}-13$ & $2.69 \mathrm{E}-14$ & $2.75 \mathrm{E}-14$ \\
\hline $\mathrm{NaI}(1) 5896$ & $1.02 \mathrm{E}-11$ & - & - & - \\
\hline NII(28) 5940/42 & $8.80 \mathrm{E}-12$ & $3.80 \mathrm{E}-14$ & $8.86 \mathrm{E}-15$ & $6.84 \mathrm{E}-15$ \\
\hline FeII(46) $5991+$ FeII(24) 6021 & $5.94 \mathrm{E}-12$ & - & - & - \\
\hline $\mathrm{CaI}(3) 6103$ & $2.46 \mathrm{E}-12$ & $3.42 \mathrm{E}-14$ & - & - \\
\hline FeII(74) 6149 & $6.35 \mathrm{E}-12$ & $2.38 \mathrm{E}-14$ & $4.11 \mathrm{E}-15$ & $3.65 \mathrm{E}-15$ \\
\hline$? 6202$ & - & - & $1.67 \mathrm{E}-15$ & $2.16 \mathrm{E}-15$ \\
\hline FeII(74) 6248 & $1.64 \mathrm{E}-12$ & - & - & - \\
\hline [OI](1) 6300 & $1.15 \mathrm{E}-11$ & $1.61 \mathrm{E}-13$ & $4.35 \mathrm{E}-14$ & $4.50 \mathrm{E}-14$ \\
\hline [OI](1) 6364 & $5.12 \mathrm{E}-12$ & $6.62 \mathrm{E}-14$ & $2.43 \mathrm{E}-14$ & $2.33 \mathrm{E}-14$ \\
\hline NII(8) 6482+FeII(74) 6456 & $6.55 \mathrm{E}-12$ & $7.19 \mathrm{E}-14$ & $1.52 \mathrm{E}-14$ & $1.34 \mathrm{E}-14$ \\
\hline $\mathrm{H} \alpha 6563+[\mathrm{NII}](1) 6584$ & $3.19 \mathrm{E}-10$ & $5.26 \mathrm{E}-12$ & $1.01 \mathrm{E}-12$ & $1.43 \mathrm{E}-12$ \\
\hline $\operatorname{HeI}(46) 6678$ & $1.02 \mathrm{E}-12$ & $3.32 \mathrm{E}-14$ & $6.71 \mathrm{E}-15$ & $8.84 \mathrm{E}-15$ \\
\hline OI(2) 6726 & $2.11 \mathrm{E}-12$ & $3.47 \mathrm{E}-14$ & - & - \\
\hline $\mathrm{OI}(21) 7002$ & $4.50 \mathrm{E}-13$ & $8.39 \mathrm{E}-15$ & - & - \\
\hline HeI(10) 7065 & $5.93 \mathrm{E}-13$ & $7.85 \mathrm{E}-14$ & $1.27 \mathrm{E}-14$ & $1.65 \mathrm{E}-14$ \\
\hline $\operatorname{CII}(20) 7115 / 20$ & $3.28 \mathrm{E}-12$ & $6.20 \mathrm{E}-14$ & - & - \\
\hline CII(3) $7231 / 36$ & $7.17 \mathrm{E}-12$ & $8.80 \mathrm{E}-14$ & $1.53 \mathrm{E}-14$ & $2.02 \mathrm{E}-14$ \\
\hline [OII](2) 7325 & $2.46 \mathrm{E}-12$ & $1.96 \mathrm{E}-13$ & $6.30 \mathrm{E}-14$ & $1.09 \mathrm{E}-13$ \\
\hline $\mathrm{NI}(3) 7424 / 42 / 69$ & $5.93 \mathrm{E}-13$ & $3.50 \mathrm{E}-14$ & - & - \\
\hline $\mathrm{OI}(55) 7477 / 9$ & $2.19 \mathrm{E}-12$ & - & - & - \\
\hline CVI 7708/26 + OIV 7713 & - & - & $9.02 \mathrm{E}-15$ & $1.13 \mathrm{E}-14$ \\
\hline OI(1) 7773 & $2.19 \mathrm{E}-11$ & $1.03 \mathrm{E}-13$ & $8.16 \mathrm{E}-15$ & $9.15 \mathrm{E}-15$ \\
\hline OI(35) $7947 / 48$ & - & $1.27 \mathrm{E}-14$ & - & - \\
\hline OI(19) 7982/87/95 & - & $4.94 \mathrm{E}-15$ & - & - \\
\hline $\operatorname{MgII}(7) 8216+\mathrm{NI}(2) 8216$ & $5.12 \mathrm{E}-12$ & $1.03 \mathrm{E}-13$ & $4.21 \mathrm{E}-15$ & $5.49 \mathrm{E}-15$ \\
\hline $\operatorname{MgII}(7) 8238+\mathrm{NI}(2) 8223$ & $7.58 \mathrm{E}-12$ & - & $4.77 \mathrm{E}-15$ & $6.67 \mathrm{E}-15$ \\
\hline OI(4) 8446 & $1.15 \mathrm{E}-10$ & $2.09 \mathrm{E}-12$ & $5.28 \mathrm{E}-14$ & $5.491 \mathrm{E}-14$ \\
\hline CaII(2) 8542 & $5.73 \mathrm{E}-12$ & - & - & - \\
\hline NI(1) $8686 / 3 / 703+\mathrm{CaII}(2) 8662$ & $5.25 \mathrm{E}-11$ & $1.122 \mathrm{E}-13$ & $7.050 \mathrm{E}-15$ & $1.396 \mathrm{E}-14$ \\
\hline NI(8) 8629 & - & $1.022 \mathrm{E}-13$ & - & - \\
\hline NI(1) 8724 & - & $5.558 \mathrm{E}-14$ & - & - \\
\hline HP 8751 & - & - & $3.754 \mathrm{E}-15$ & $5.122 \mathrm{E}-15$ \\
\hline HP 8863 & - & $4.604 \mathrm{E}-14$ & $5.240 \mathrm{E}-15$ & $8.226 \mathrm{E}-15$ \\
\hline$? 8930$ & - & - & $1.159 \mathrm{E}-15$ & $2.686 \mathrm{E}-15$ \\
\hline HP 9015 & - & $5.89 \mathrm{E}-14$ & $6.184 \mathrm{E}-15$ & $1.117 \mathrm{E}-14$ \\
\hline HP 9229 & - & $1.51 \mathrm{E}-13$ & $2.137 \mathrm{E}-14$ & $3.139 \mathrm{E}-14$ \\
\hline $\mathrm{NI}(7) 9393+\mathrm{CI}(9) 9406$ & - & $9.93 \mathrm{E}-14$ & - & - \\
\hline
\end{tabular}

where $\tau$ is the optical depth of the line $\lambda 6300$ as derived by solving the equation:

$\frac{F_{\lambda 6300}}{F_{\lambda 6364}}=\frac{1-\mathrm{e}^{-\tau}}{1-\mathrm{e}^{-\tau / 3}}$

and $F_{\lambda(i)}$ is the observed (unreddened) line flux at the specified wavelength. The OI mass can be derived knowing that the line flux is proportional to the mass of the emitting region (the optical depth is not large) scaled by the square of the distance, $d$, i.e. (again, after substitution of the tabulated constants):

$$
\begin{aligned}
M_{\mathrm{OI}}= & 152 \times d_{\mathrm{kpc}}^{2} \exp \left(\frac{22850}{T_{\mathrm{e}}}\right) \\
& \times 10^{1.05 E(B-V)} \frac{\tau}{1-\mathrm{e}^{-\tau}} F_{\lambda 6300} M_{\odot} .
\end{aligned}
$$

The measured line flux ratios and the derived gas temperature and mass are reported in Table 7 . We found that the gas temperature, $T_{\mathrm{e}}$, is within the range $3500-5000 \mathrm{~K}$ for both 
Table 6. Nova LMC 2002 emission lines fluxes. Flux measurements are affected by errors of he order of $\sim 30 \%$.

\begin{tabular}{|c|c|c|c|}
\hline Line & "09-03-2002 & "14-03-2002 & "11-08-2002 \\
\hline H11(2) 3771 & $1.03 \mathrm{E}-12$ & $4.40 \mathrm{E}-13$ & - \\
\hline H10(2) 3798 & $4.80 \mathrm{E}-13$ & $2.58 \mathrm{E}-13$ & - \\
\hline $\mathrm{H} \eta 3835+\operatorname{MgI}(3) 3835$ & $8.34 \mathrm{E}-13$ & $4.91 \mathrm{E}-13$ & - \\
\hline $\mathrm{H} \zeta 3889$ & $6.24 \mathrm{E}-13$ & $3.19 \mathrm{E}-13$ & - \\
\hline CaII(1) 3934 & $2.34 \mathrm{E}-12$ & $1.08 \mathrm{E}-12$ & - \\
\hline $\mathrm{H} \epsilon 3970+\mathrm{CaII}(1) 3969$ & $2.32 \mathrm{E}-12$ & $7.95 \mathrm{E}-13$ & $9.40 \mathrm{E}-16$ \\
\hline [SII](1) 4069 & - & - & $1.59 \mathrm{E}-15$ \\
\hline ? 4091 & - & - & $5.65 \mathrm{E}-15$ \\
\hline $\mathrm{H} \delta 4102$ & $2.60 \mathrm{E}-12$ & $8.24 \mathrm{E}-13$ & $5.80 \mathrm{E}-15$ \\
\hline$[\mathrm{FeII}](21) 4177$ & - & - & $7.33 \mathrm{E}-16$ \\
\hline FeII(27) 4176+(28) 4179 & $9.11 \mathrm{E}-13$ & $4.53 \mathrm{E}-13$ & - \\
\hline OII(36) 4190/86 & - & - & $6.33 \mathrm{E}-16$ \\
\hline FeII(27) 4233 & $7.01 \mathrm{E}-13$ & $4.56 \mathrm{E}-13$ & - \\
\hline CII(6) 4267 & - & - & $8.92 \mathrm{E}-16$ \\
\hline FeII(27) 4273 & $4.51 \mathrm{E}-13$ & $5.62 \mathrm{E}-13$ & - \\
\hline FeII(27) $4303+(28) 4297$ & $1.10 \mathrm{E}-12$ & $6.70 \mathrm{E}-13$ & - \\
\hline $\mathrm{H} \gamma 4341$ & $4.30 \mathrm{E}-12$ & $2.19 \mathrm{E}-12$ & $4.72 \mathrm{E}-15$ \\
\hline [OIII](2) 4363 & - & - & $1.94 \mathrm{E}-14$ \\
\hline FeII(27) 4417 & $5.47 \mathrm{E}-13$ & $3.33 \mathrm{E}-13$ & - \\
\hline OII(5) 4452 & $3.38 \mathrm{E}-13$ & $1.51 \mathrm{E}-13$ & - \\
\hline FeII(37) 4489/91 & $5.54 \mathrm{E}-13$ & $4.55 \mathrm{E}-13$ & - \\
\hline FeII(38) $4523+(37) 4520 / 34$ & $6.68 \mathrm{E}-13$ & $6.72 \mathrm{E}-13$ & - \\
\hline FeII(37) $4556+(38) 4550$ & $1.22 \mathrm{E}-12$ & $6.93 \mathrm{E}-13$ & - \\
\hline FeII(38) $4596+$ OII(5) 4591 & $8.57 \mathrm{E}-13$ & $6.850 \mathrm{E}-13$ & - \\
\hline NII(5) 4607/14/21 & - & - & $1.60 \mathrm{E}-15$ \\
\hline NIII(2) 4640 & $6.78 \mathrm{E}-13$ & $4.82 \mathrm{E}-13$ & $7.21 \mathrm{E}-15$ \\
\hline HeII(1) 4686 & - & - & $2.36 \mathrm{E}-15$ \\
\hline $\mathrm{H} \beta 4861$ & $9.52 \mathrm{E}-12$ & $6.41 \mathrm{E}-12$ & $1.32 \mathrm{E}-14$ \\
\hline FeII(42) 4924 & $1.79 \mathrm{E}-12$ & $1.43 \mathrm{E}-12$ & - \\
\hline [OIII](1) 4959 & - & - & $2.79 \mathrm{E}-14$ \\
\hline [OIII](1) 5007 & - & - & $8.31 \mathrm{E}-14$ \\
\hline FeII(42) 5018 & $1.64 \mathrm{E}-12$ & $1.50 \mathrm{E}-12$ & - \\
\hline $\operatorname{HeI}(47) 5048$ & $5.07 \mathrm{E}-13$ & $5.25 \mathrm{E}-13$ & - \\
\hline FeII(42) 5169 & $1.78 \mathrm{E}-12$ & $1.29 \mathrm{E}-12$ & - \\
\hline NII(66) 5176/80 & - & - & $3.24 \mathrm{E}-16$ \\
\hline FeII(49) 5198 & $1.06 \mathrm{E}-12$ & $5.485 \mathrm{E}-13$ & - \\
\hline FeII(49) $5235+(48)$ & $8.50 \mathrm{E}-13$ & $4.58 \mathrm{E}-13$ & - \\
\hline FeII(49) $5276+(48)$ & $1.16 \mathrm{E}-12$ & $7.05 \mathrm{E}-13$ & - \\
\hline FeII(49) $5317+(48)$ & $1.47 \mathrm{E}-12$ & $9.42 \mathrm{E}-13$ & - \\
\hline FeII(55) 5535 & $4.87 \mathrm{E}-13$ & $3.60 \mathrm{E}-13$ & - \\
\hline [OI](3) 5577 & $5.78 \mathrm{E}-13$ & $5.14 \mathrm{E}-13$ & $6.75 \mathrm{E}-16$ \\
\hline NII(3) 5667/80 & $4.62 \mathrm{E}-13$ & $5.050 \mathrm{E}-13$ & $1.47 \mathrm{E}-15$ \\
\hline [NII](3) 5755 & $3.55 \mathrm{E}-13$ & $3.66 \mathrm{E}-13$ & $1.50 \mathrm{E}-14$ \\
\hline CIV(1) 5805 & $3.13 \mathrm{E}-13$ & $2.29 \mathrm{E}-13$ & $7.28 \mathrm{E}-16$ \\
\hline HeI(11) 5876 & - & - & $1.71 \mathrm{E}-15$ \\
\hline $\mathrm{NaI}(\mathrm{D}) 5896$ & $7.88 \mathrm{E}-13$ & $5.85 \mathrm{E}-13$ & - \\
\hline NII(28) 5940/42 & - & - & $6.47 \mathrm{E}-16$ \\
\hline FeII(74) $6147+$ NII(36/60) 6168 & $8.23 \mathrm{E}-13$ & $5.32 \mathrm{E}-13$ & - \\
\hline FeII(74) 6248 & $4.95 \mathrm{E}-13$ & $3.36 \mathrm{E}-13$ & - \\
\hline
\end{tabular}

novae (cf. Williams 1994, his Table 2). The OI mass was computed assuming the distances of the LMC and SMC to be $51.4 \mathrm{kpc}$ (Panagia 1998) and $56.4 \mathrm{kpc}$, respectively, the latter value resulting from the average of recent distance measurements (Harries et al. 2003; Dolphin et al. 2001; Bono et al. 2001; and Cioni et al. 2000). We derived OI masses of the 
Table 6. continued.

\begin{tabular}{|c|c|c|c|}
\hline Line & 09-03-2002 & 14-03-2002 & 111-08-2002 \\
\hline$[\mathrm{OI}](1) 6300$ & $6.29 \mathrm{E}-13$ & $7.07 \mathrm{E}-13$ & $6.95 \mathrm{E}-15$ \\
\hline [OI](1) 6364 & $4.03 \mathrm{E}-13$ & $3.29 \mathrm{E}-13$ & $2.80 \mathrm{E}-15$ \\
\hline FeII(74) 6417 & $2.53 \mathrm{E}-13$ & - & - \\
\hline FeII(74) 6456 & $6.92 \mathrm{E}-13$ & $3.546 \mathrm{E}-13$ & - \\
\hline NII(8) 6482 & - & - & $1.37 \mathrm{E}-15$ \\
\hline $\mathrm{H} \alpha+[\mathrm{NII}](1) 6584$ & $2.88 \mathrm{E}-11$ & $2.83 \mathrm{E}-11$ & $8.33 \mathrm{E}-14$ \\
\hline $\operatorname{HeI}(46) 6678$ & - & - & $4.69 \mathrm{E}-16$ \\
\hline HeI(10) 7065 & - & - & $1.32 \mathrm{E}-15$ \\
\hline CII(20) $7115 / 9$ & $1.96 \mathrm{E}-12$ & $7.85 \mathrm{E}-13$ & - \\
\hline CII(3) 7234 & $7.75 \mathrm{E}-13$ & $6.32 \mathrm{E}-13$ & $2.06 \mathrm{E}-15$ \\
\hline$[\mathrm{OII}](2) 7319 / 31$ & $3.35 \mathrm{E}-13$ & $7.93 \mathrm{E}-14$ & $1.32 \mathrm{E}-14$ \\
\hline $\mathrm{OI}(55) 7477 / 79 / 81+\mathrm{NI}(3) 7424 / 42 / 68$ & $1.76 \mathrm{E}-12$ & $4.72 \mathrm{E}-13$ & - \\
\hline CIV 7726 & - & - & $1.67 \mathrm{E}-15$ \\
\hline OI(1) 7773 & $5.66 \mathrm{E}-12$ & $3.22 \mathrm{E}-12$ & $5.71 \mathrm{E}-16$ \\
\hline $\operatorname{MgII}(8) 7877 / 86$ & $1.08 \mathrm{E}-12$ & - & - \\
\hline OI(34) 8227 & $7.20 \mathrm{E}-13$ & $4.73 \mathrm{E}-12$ & - \\
\hline $\mathrm{CaII}(13) 8250$ & $1.33 \mathrm{E}-12$ & $6.68 \mathrm{E}-13$ & - \\
\hline HP(11) $8346+\mathrm{CI}(10) 8335$ & $1.10 \mathrm{E}-12$ & $4.55 \mathrm{E}-13$ & - \\
\hline OI(4) $8446 / 7$ & $4.94 \mathrm{E}-12$ & $4.77 \mathrm{E}-12$ & $2.35 \mathrm{E}-15$ \\
\hline CaII(2) 8498 & $3.08 \mathrm{E}-12$ & $1.977 \mathrm{E}-12$ & - \\
\hline CaII(2) 8542 & $2.85 \mathrm{E}-12$ & $2.05 \mathrm{E}-12$ & - \\
\hline CaII(2) 8662 & $2.18 \mathrm{E}-12$ & $1.56 \mathrm{E}-12$ & - \\
\hline [CI] 8727 & $2.71 \mathrm{E}-12$ & $1.49 \mathrm{E}-12$ & - \\
\hline HP(9) 8863 & $1.16 \mathrm{E}-12$ & $5.71 \mathrm{E}-13$ & - \\
\hline NI(15) 9061 & $2.95 \mathrm{E}-12$ & $1.10 \mathrm{E}-12$ & \\
\hline CI(3) 9087 & $5.40 \mathrm{E}-12$ & $2.86 \mathrm{E}-12$ & - \\
\hline $\operatorname{MgII}(1) 9226$ & $1.73 \mathrm{E}-12$ & $1.12 \mathrm{E}-12$ & - \\
\hline OI(8) 9264 & $2.47 \mathrm{E}-12$ & $1.13 \mathrm{E}-12$ & - \\
\hline CI(9) 9406 & $2.91 \mathrm{E}-12$ & $2.03 \mathrm{E}-12$ & - \\
\hline HP(8) 9546 & $7.92 \mathrm{E}-13$ & $5.39 \mathrm{E}-13$ & - \\
\hline CI(2) 9627 & $1.39 \mathrm{E}-12$ & $7.87 \mathrm{E}-13$ & - \\
\hline CI(2) 9658 & $1.81 \mathrm{E}-12$ & $8.59 \mathrm{E}-13$ & - \\
\hline HP(8) 10049 & $1.89 \mathrm{E}-12$ & $1.33 \mathrm{E}-12$ & - \\
\hline NI(18) 10117 & $2.14 \mathrm{E}-12$ & $1.29 \mathrm{E}-12$ & - \\
\hline
\end{tabular}

order of a few $10^{-5} M_{\odot}$ (see our Table 7 and Table 3 of Williams 1994).

\subsection{The HII mass and the filling factor}

We estimate the mass of the ionized hydrogen and its filling factor, following the approximation described in Mustel \& Boyarchuk (1970) ${ }^{1}$. The volume of the ejecta can be approximated by a spherical shell of radius $r=v_{\exp } \Delta t$ (where $v_{\exp }$ is the expansion velocity measured from the spectra and $\Delta t$ is the time since maximum), and thickness $f r$, with $f$ given by the ratio of the gas thermal velocity over the expansion velocity, i.e. $v_{\text {therm }} / v_{\text {exp }}$. The volume is furthermore corrected by a factor $\varepsilon \leq 1$, the filling factor, which parametrizes the clumpiness of the ejecta. Thus:

$V=4 \pi\left(v_{\exp } \Delta t\right)^{3} f \varepsilon$

${ }^{1}$ We used the $\mathrm{H} \beta$ flux and not the $\mathrm{H} \alpha$ flux as in Mustel \& Boyarchuk (1970), due to the heavy blend of the $\mathrm{H} \alpha$ and [NII] lines.
The observed line flux is the emitted flux reduced by the inverse square effect of the geometrical dilution, thus:

$I_{\lambda}=\left[\left(\frac{4 \pi j_{\lambda}}{N_{\mathrm{e}} N_{\mathrm{p}}}\right) N_{\mathrm{e}} N_{\mathrm{p}} V\right] \frac{1}{4 \pi d^{2}}$

where $I_{\lambda}$ is the measured line flux, $j_{\lambda}$ is the gas emissivity, the factor $4 \pi j_{\lambda} /\left(N_{\mathrm{p}} N_{\mathrm{e}}\right)$ is tabulated in Osterbrock (1989), $N_{\mathrm{p}}\left(N_{\mathrm{e}}\right)$ is the proton (electron) density, and $d$ is the distance. Knowing the volume, the electron density of the ejecta, and assuming $N_{\mathrm{p}} \sim N_{\mathrm{e}}$, we can compute the HII mass; furthermore, combining the Eqs. (4) and (5) we can derive the filling factor. Our spectra do not show any of the typical lines used as a diagnostic for the electron density (see Osterbrock 1989), thus, we have proceeded according to Filippenko \& Halpern (1984). Using the observed [OIII] line ratio $(4959+5007) / 4363$, we derived the electron density, $N_{\mathrm{e}}$, corresponding to the typical nebular temperature of $\sim 10^{4} \mathrm{~K}$. We found that a density of $N_{\mathrm{e}} \sim 10^{7} \mathrm{~cm}^{-3}$ is required in both the Nova SMC March 2002 spectrum and Nova LMC August 2002 spectrum. In order to 


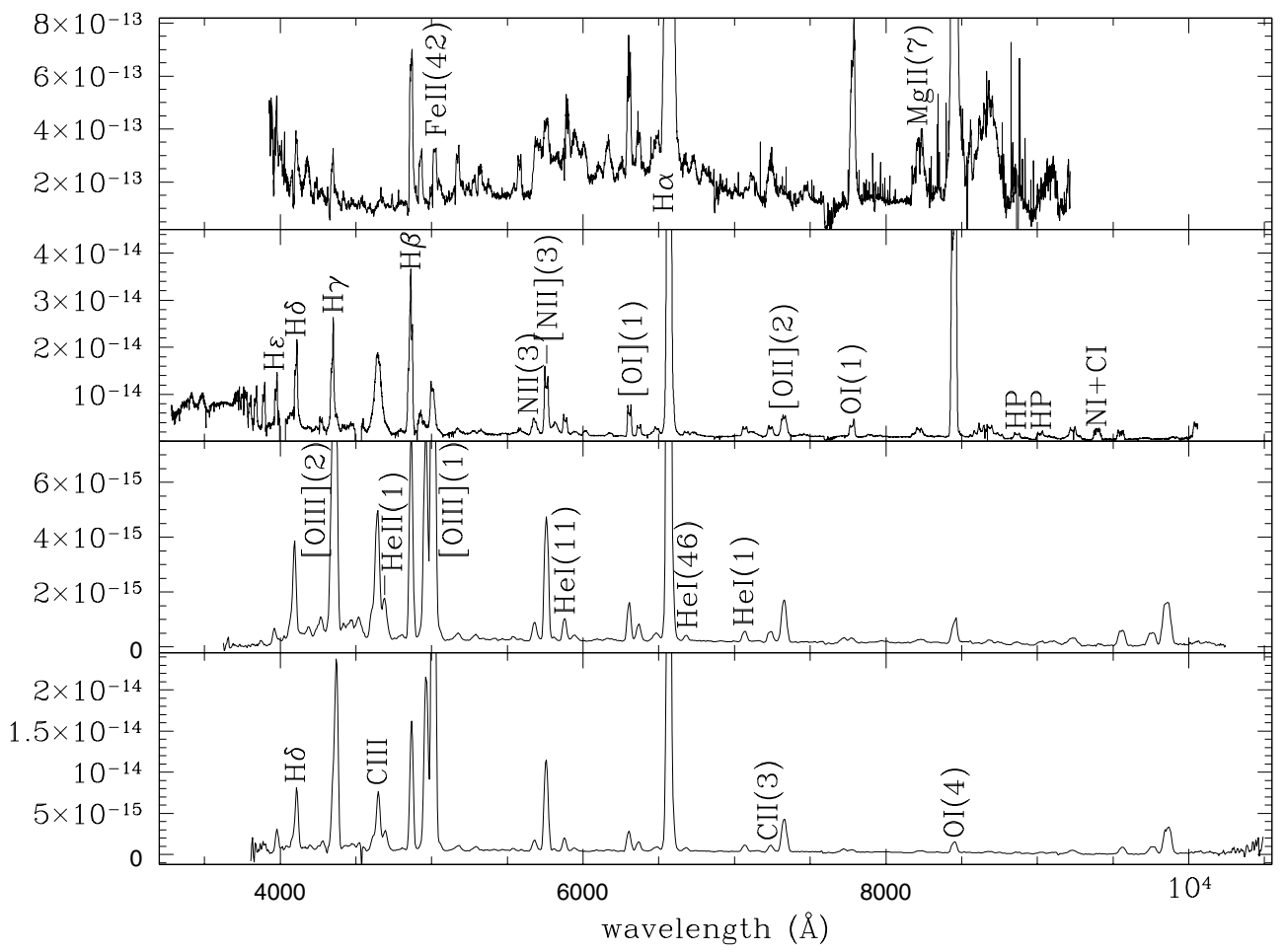

Fig. 2. Nova SMC 2001. From top to bottom: November 2001, December 2001, February 2002, and March 2002 spectra. Flux units are erg s $\mathrm{sm}^{-1} \AA^{-1}$.
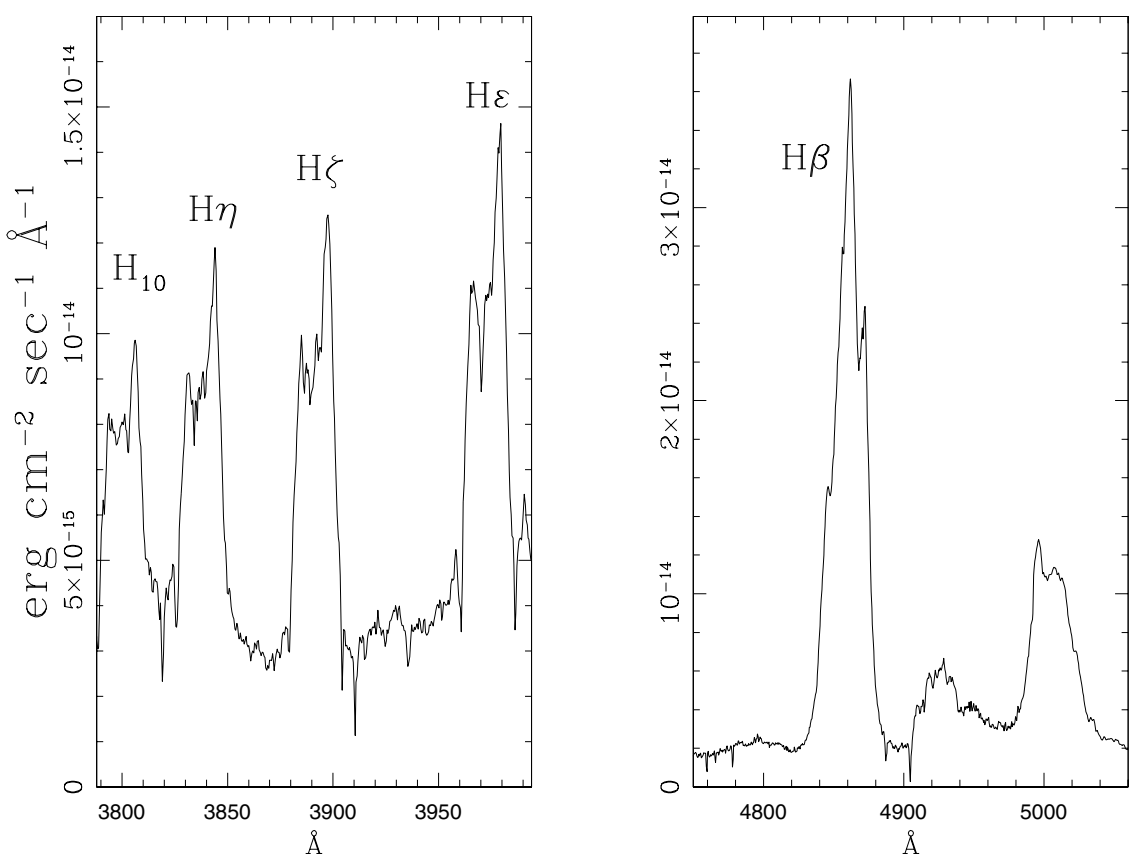

Fig. 3. Zoomed-in view of Nova SMC 2001 December spectrum. The two panels show the different profiles of the Balmer lines (see also Sect. 3.1).

obtain temperature values which are close to those reported in Table 7 (namely $3500 \div 5000 \mathrm{~K}$ ), we would need input densities higher than $10^{8} \mathrm{~cm}^{-3}$. However, such high densities are hardly compatible with nebular regimes. In addition, the observed [OI] $\lambda 6300 / \lambda 6364$ line ratio (Table 7, Col. 3 ) is definitely smaller than the theoretical value of $\sim 3$, expected at nebular regimes (Osterbrock 1989). This suggests that the [OI] and the Balmer+[OIII] emissions arise from different ejected layers. Williams (1994) has explained this behavior (exhibited by most novae) by assuming that the optically thick lines of [OI] originate in cool, high density blobs $\left(N(\mathrm{H})>10^{14} \mathrm{~cm}^{-3}\right)$ of neutral material embedded within the ejected shell. The filling factor measurements $\varepsilon \sim 10^{-4} \div 10^{-1}$ (Table 8, Col. 6) lend support to this view, thus suggesting that both Nova SMC 2001 


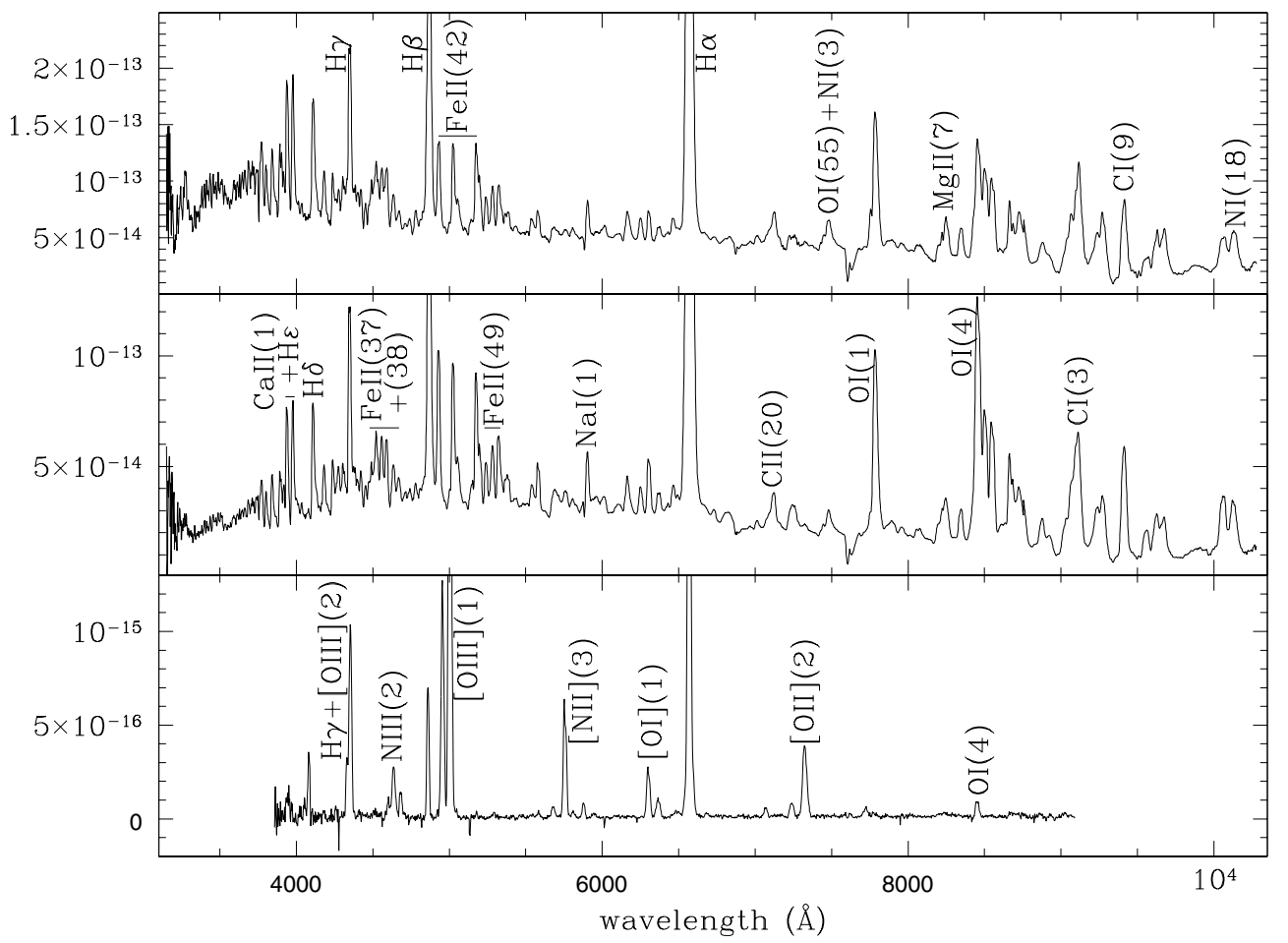

Fig. 4. Nova LMC 2002 spectra. From top to bottom: March 9, March 14, and August 8 spectra. Flux units are $\operatorname{erg~s}^{-1} \mathrm{~cm}^{-2} \AA^{-1}$.

Table 7. The OI line flux ratio, the corresponding opacity $\tau_{\lambda 6300}, T_{\mathrm{e}}$, and mass derived for each nova at each epoch according to Williams (1994). See text for more details.

\begin{tabular}{cccccc}
\hline \hline Object & Obs. date & $r=\frac{F_{16300}}{F_{\lambda 6364}}$ & $\tau_{\lambda 6300}$ & $T_{\mathrm{e}}(\mathrm{K})$ & $M_{\mathrm{OI}}\left(M_{\odot}\right)$ \\
\hline Nova SMC 2001 & $01 / 11 / 01$ & 2.24 & 0.98 & 4900 & $9.83 \mathrm{E}-4$ \\
& $06 / 12 / 01$ & 2.43 & 0.68 & 4600 & $1.89 \mathrm{E}-5$ \\
& $17 / 02 / 02$ & 1.79 & 1.96 & 3500 & $4.30 \mathrm{E}-5$ \\
Nova LMC 2002 & $04 / 03 / 02$ & 1.93 & 1.60 & 3600 & $3.28 \mathrm{E}-5$ \\
& $09 / 03 / 02$ & 1.56 & 2.75 & 5200 & $7.96 \mathrm{E}-5$ \\
& $14 / 03 / 02$ & 2.15 & 1.14 & 5600 & $3.82 \mathrm{E}-5$ \\
& $11 / 08 / 02$ & 2.48 & 0.61 & 4000 & $1.46 \mathrm{E}-6$ \\
\hline
\end{tabular}

Table 8. The observed [OIII] line ratio and the computed filling factor, $\varepsilon$, and hydrogen mass. The spectra used are those taken in March 2002 and August 2002 for Nova SMC 2001 and Nova LMC 2002, respectively. See Sect. 3.3 for more details.

\begin{tabular}{ccccccc}
\hline \hline Object & $(4959+5007) / 4363$ & $N_{\mathrm{e}}$ & $T_{\mathrm{e}}$ & $f$ & $\varepsilon$ & $M_{\mathrm{H}}\left(M_{\odot}\right)$ \\
\hline Nova SMC 2001 & 3.65 & $10^{7}$ & 12000 & 0.41 & 0.16 & $6.07 \mathrm{E}-5$ \\
& & $10^{8}$ & 8500 & 0.34 & 0.002 & $5.02 \mathrm{E}-6$ \\
Nova LMC 2002 & 5.69 & $10^{7}$ & 10000 & 0.45 & 0.01 & $2.38 \mathrm{E}-6$ \\
& & $10^{8}$ & 7600 & 0.39 & $1.0 \mathrm{E}-4$ & $2.0 \mathrm{E}-7$ \\
\hline
\end{tabular}

and Nova LMC 2002 have clumpy ejecta rather than shells homogeneously filled in.

\subsection{The absolute magnitudes}

The study of the distribution of the absolute magnitude of novae at maximum in the MC has a specific interest. Indeed, several authors (e.g., van den Bergh \& Pritchet 1986; Capaccioli et al. 1989) have already pointed out the existence of a subclass of superluminous classical novae which are systematically brighter (by about $1 \mathrm{mag}$ ) than predicted by the general MMRD (Maximum Magnitude vs. Rate of Decline) relation (Della Valle \& Livio 1995). The last example of such an object was the exceptionally bright Nova LMC 1991 (Della Valle 1991). The frequency of occurrence of these events is very uncertain and its estimate is of interest: on one hand they are potential "caveats" for using the Novae as distance indicators in very distant galaxies (e.g., Pritchet \& van den Bergh 1987; 
Table 9. LMC novae in the literature.

\begin{tabular}{|c|c|c|c|c|}
\hline Nova & Spectral description & Spectral class & $t 2$ & Ref. \\
\hline LMC 2002 & $\begin{array}{l}H W Z I \sim 2150, \text { FeII and low } \\
\text { ionization elements }\end{array}$ & FeII & 12 & this paper \\
\hline LMC $1995^{\dagger}$ & $\begin{array}{l}\text { P-Cyg profiles }(900 \text { and } \\
\left.1500 \mathrm{~km} \mathrm{~s}^{-1}\right), \mathrm{Fe} \text { and } \mathrm{Na} \text { emis- } \\
\text { sion lines early dust formation }\end{array}$ & FeII & 7 & 1,2 \\
\hline LMC 1992 & $\begin{array}{l}\text { very narrow emission lines, } \\
\text { P-Cyg profiles, } \mathrm{Na} \text { and } \mathrm{Fe}\end{array}$ & FeII & 6 & 3 \\
\hline LMC 1991 & $F W H M 2300 \mathrm{~km} \mathrm{~s}^{-1}$ & FeII & 6 & 4,5 \\
\hline LMC 1990 \#2 & $\begin{array}{l}\text { strong } \mathrm{HeI} \text { and HeII lines, } \\
F W H M \sim 5500 \mathrm{~km} \mathrm{~s}^{-1} \text {, mimic } \\
1968 \text { outburst and RN U Sco } \\
\text { and V394 CrA }\end{array}$ & $\mathrm{He} / \mathrm{N}$ & 3 & 6,7 \\
\hline LMC 1990 \#1 & $\begin{array}{l}\text { very broad emission lines } \\
\left(F W H M \sim 5600 \mathrm{~km} \mathrm{~s}^{-1}\right), \\
\mathrm{UV} \text { maximum spectrum very } \\
\text { similar to V693 CrA and } \\
\mathrm{U} \text { Sco. Early development of } \\
\text { HeI and HeII emission lines as } \\
\text { well as [Ne]. }\end{array}$ & $\mathrm{He} / \mathrm{N}$ & 4.5 & 8,9 \\
\hline LMC $1988 \# 2$ & $\begin{array}{l}\text { broad Balmer and FeII emis- } \\
\text { sion lines, P-Cyg profiles, } \\
F W H M \sim 3500 \mathrm{~km} \mathrm{~s}^{-1}\end{array}$ & FeIIb & 5 & 10,11 \\
\hline LMC $1988 \# 1$ & $\begin{array}{l}\text { FeII, NaI, OI and CaII with } \\
\text { P-Cyg profiles }\end{array}$ & FeII & 20 & 10,12 \\
\hline LMC $1981^{\dagger \dagger}$ & $\begin{array}{l}\text { very fast and similar to } \\
\text { V1500 Cyg }\end{array}$ & FeIIb & 3 & 13 \\
\hline LMC 1978 \#1 & $\begin{array}{l}\text { narrow Balmer lines, normal } \\
\text { past maximum spectrum, fast } \\
\text { decline }\end{array}$ & FeII & 8 & 14,15 \\
\hline
\end{tabular}

References: IAUC 6143 (1); AAVSO interactive light curve (2); IAUC 5651, 5653, 5656, 5657, 5659, 5661, 5669,5683 (3); IAUC 5260 (4); Schwartz et al. (2001) (5); IAUC 4964, 4975 (6); Sekiguchi et al. (1990) (7); IAUC 4946, 4949, 4956, 4960, 4961, 4964 (8); Vanlandingham et al. (1999) (9); Williams et al. (1991) (10); IAUC 4663, 4664, 4666, 4670, 4673 (11); IAUC 4568, 4569, 4574, 4577, 4580, 4585, 4588, 4589, 4601, 4610 (12); IAUC 3648, 3641 (13), IAUC 3206 (14), Capaccioli et al. (1990) (15).

$\dagger$ Nova LMC 1995's $t_{2}$ is extrapolated assuming linear rate of decline from IAUC data points covering the first 4 days. A similar value may possibly be inferred from AAVSO unvalidated data points on line.

${ }^{\dagger \dagger}$ The $t_{2}$ for nova LMC 1981 is really unknown: Duerbeck (1981, IAUC 3641) reports that the nova is very fast and that the spectrum taken few days after discovery strongly resemble the spectra of CP Pup and V1500 Cyg +4.5 mag from maximum. In such a hypothesis we assume $t_{2} \sim 3-5$ as given in the table above. On the other side Maza (1981, IAUC 3641) confirming the discovery of the nova after 6 days from the first announcement reports a decline of $+0.7 \mathrm{mag}$. As rate of decline is roughly linear within 2 or 3 mag from maximum, and assuming the decline rate given by Maza the nova should have a $t_{2}$ of $\sim 18 \mathrm{dd}$.

Della Valle \& Gilmozzi 2002). On the other hand they can play an important role in understanding the nature of the nova phenomenon (see Schwarz et al. 2001). In the case of Nova LMC 2002, both $t_{2}=12$ days and the apparent magnitude at maximum, $m_{V}=10.4 \mathrm{mag}$, are consistent with the values of a "normal" nova. Furthermore, we have in hand enough parameters to provide an independent check. From $t_{2}=12$ days, one infers an absolute magnitude at maximum of $M_{V}=-8.6$ through Della Valle \& Livio's (1995) MMRD relation. We then used the observed apparent maximum magnitude, $m_{V}=10.4 \mathrm{mag}$, and the LMC distance of $51.4 \mathrm{kpc}$ (Panagia 1998), to solve the equation of the distance modulus for $A_{\mathrm{V}}$. We have found $A_{\mathrm{V}}=0.42$ and $E(B-V)=0.14$, in excellent agreement with the average $\langle E(B-V)\rangle=0.13$, derived independently and adopted through this paper.
Nova SMC 2001 was discovered during the early decline, thus we cannot repeat the above cross-check, but rather work out the nova absolute magnitude in a different way. Taking advantage of the similarities between nova SMC 2001 and nova LMC 1988 No. 1 (see Sect. 1), we can assume $t_{2}<$ 20 days for nova SMC 2001 (see also Table 9). This implies that the nova was about 1 mag below maximum at the time of Liller's discovery. The absolute maximum magnitude of Nova SMC 2001 is thus constrained within the range $-8.3<$ $M_{V}<-7.7 \mathrm{mag}$.

\subsection{The nova populations}

We collected from the literature all the photometric and spectroscopic data for LMC novae in order to derive the $t_{2}$ from 


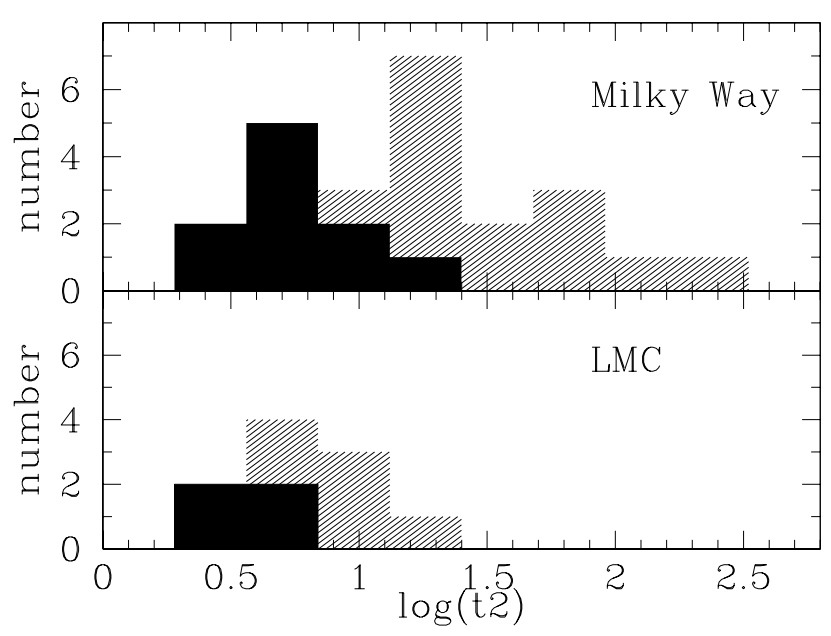

Fig. 5. Histogram of the classical nova distribution versus decline speed for the Galaxy (top panel) and the LMC (bottom panel). Dark area represents the He/N and FeIlb classes; shaded area is for FeII novae. See text for discussion.

the light curves and the spectroscopic classification according to the Cerro Tololo scheme (see Table 9). We used the data reported in Table 9 together with those in Tables 1 and 2 of Della Valle \& Livio (1998), to plot the frequency distribution (Fig. 5) of the rates of decline for the novae in the LMC and the Milky Way (Fig. 5, lower and upper panel, respectively).

The distribution of the nova population in the Milky Way is bimodal, and consists of fast declining objects $(\log (t 2)<1.1)$ originating mostly in the disk and slow declining novae forming also in the bulge/thick disk (Della Valle et al. 1992; Della Valle \& Livio 1998). The observed LMC novae distribution (bottom panel of Fig. 5) matches the disk population within the Milky Way (top panel of Fig. 5). This is an expected result since the LMC is a bulge-less galaxy. Moreover, Subramaniam \& Anupama (2002), studying the parent population of the novae in the LMC, found that LMC classical novae are surrounded by an intermediate age population within the range $3.2 \div 1.0$ Gyr. Subramaniam \& Anupama (2002) studied also the parent population around Nova SMC 1994 and found that Nova SMC 1994, which has the same spectroscopic evolution as Nova SMC 2001 (see Sect. 4), is surrounded by an old population $(1 \div 10 \mathrm{Gyr})$ similar to the two slow novae observed in the LMC.

\section{Summary and conclusions}

In this paper we have presented the early-decline/middle-stages spectra of two recent novae discovered in the Magellanic clouds: Nova SMC 2001 and Nova LMC 2002.

Nova SMC 2001 is the first nova in the SMC that has been spectroscopically observed during the early decline. This object displays a classical FeII-type spectrum, characterized by moderately high expansion velocities (FWHM $1900 \mathrm{~km} \mathrm{~s}^{-1}$ ). The observations obtained during the nebular phase indicate that Nova SMC 2001 developed a standard nebular spectrum (Williams 1992). The auroral-nebular phase of Nova SMC 2001 is similar to that exhibited by Nova SMC 1994, the only other SMC nova which has been spectroscopically observed (though only during its nebular phase, de Laverny et al. 1998).

Nova LMC 2002 is characterized by a rapid photometric evolution, $t_{2} \sim 12$ days, and a relatively high expansion velocity $\left(H W Z I=2150 \mathrm{~km} \mathrm{~s}^{-1}\right)$. Both aspects make this nova a borderline object between the fast and slow nova classes defined by Della Valle \& Livio (1998). The spectroscopic evolution shows a typical FeII type object and our latest observation suggests the development of a standard nebular spectrum.

Spectra of the two novae were analyzed to provide emission line fluxes, measurements of the OI and HII ejected masses (Tables 7 and 8), and estimates of the filling factor (Table 8). We obtained masses of the order of a few $\times 10^{-5} M_{\odot}$ and $\sim 10^{-6} M_{\odot}$, for the OI and the HII, respectively. After assuming that the $\mathrm{O}$ mass represents $\sim 15 \%$ of the total mass of the shell (Williams 1994; Gehrz et al. 1993), we can infer that the mass of the ejecta is of the order of $2 \div 3 \times 10^{-4} M_{\odot}$. We estimated the filling factor of the ejecta to be in the range $\varepsilon \sim 10^{-4} \div 10^{-1}$. In spite of the rough assumptions used in our computations, the derived values are consistent with the estimate $\left(\varepsilon \sim 10^{-5} \div 10^{-2}\right)$ provided by Shara et al. (1997), after studying the ejecta of the recurrent nova T Pyx on HST images.

The intensity ratio [OI] $\lambda 6300 / \lambda 6364$ shows an anomalous value of $r \sim 2$ rather than $\sim 3$ as expected on theoretical grounds. We note that such an anomalous ratio is commonly observed in Milky Way novae (e.g. Nova V382 Vel 1999; Della Valle et al. 2002). The optically thick [OI] lines are likely formed in high density blobs of neutral material (see Williams 1994, for a discussion), thus suggesting a clumpy structure of the nova ejecta. A clumpy ejecta is also suggested by the values $\varepsilon \ll 1$ reported above.

Our results indicate that some of the general physical properties of LMC and SMC novae are comparable to those of galactic novae (e.g. spectral evolution, clumpiness of the ejecta, etc). The main difference between the galactic and LMC nova population is in the distribution of $t_{2}$ (see Fig. 5). The LMC nova population is formed by bright and fast novae, whereas the faint-slow component, which is observed in the Milky Way, is completely missing. This behavior is not due to an observational bias (see Della Valle 2002, for a discussion) and it can be explained, at least in part, by theoretical models (Starrfield et al. 1998, 2000), which predict a larger brightness and a larger amount of accreted mass at low metallicity regimes.

\section{References}

Bessel, M. S. 1991, A\&A, 242, L17

Bono, G., Caputo, F., \& Marconi, M. 2001, MNRAS, 325, 1353

Bosch, G. L., Barba, R. H., \& Morell, N. I. 2001, IAUC, No. 7744

Capaccioli, M., Della Valle, M., Rosino, L., \& D’Onofrio, M. 1989, AJ, 97, 1622

Capaccioli, M., Della Valle, M., D’Onofrio, M., \& Rosina, L. 1990, ApJ, 360, 63

Cioni, M.-R. C., van der Marel, R. P., Loup, C., \& Habing, H. J. 2000, A\&A, 359, 601

de Laverny, P., Beaulieu, J. P., Asplund, M., et al. 1998, A\&A, 335, L93

Della Valle, M. 1991, A\&A, 252, L9 
Della Valle, M. 2002, Classical nova explosions, AIP Conf. Proc., 637, 443

Della Valle, M., \& Livio, M. 1995, ApJ, 452, 704

Della Valle, M., \& Livio, M. 1998, ApJ, 506, 818

Della Valle, M., \& Gilmozzi, R. 2002, Science, 296, 1275

Della Valle, M., Bianchini, A., Livio, M., \& Orio, M. 1992, A\&A, 266, 232

Della Valle, M., Rosino, L., Bianchini, A., \& Livio, M. 1994, A\&A, 287,403

Della Valle, M., Saviane, I., \& Williams, R. E. 2001, IAUC, No. 7743

Della Valle, M., Pasquini, L., Daou, D., \& Williams, R. E. 2002, A\&A, 390,155

Dolphin, A. E., Walker, A. R., Hodge, P. W., et al. 2001, ApJ, 562, 303

Duerbeck, H. W. 1990, in Physics of Classical Novae, ed. A. Cassatella, \& R. Viotti (Berlin: Springer), IAU Coll., 122, 34

Dutra, C. M., Bica, E., Clariá, J. J., Piatti, A. E., \& Ahumada, A. V. 2001, A\&A, 371, 895

Filippenko, A. V., \& Halpern, J. P. 1984, ApJ, 285, 474

Gehrz, R. D., Truran, J. W., \& Williams, R. E. 1993, in Protostar and planets III, ed. E. H. Levy, \& J. I. Lunine (Tucson: Univ. Arizona Press), 75

Gilmore, A. C. 2002, IAUC, No. 7853

Hack, M., Salvelli, P. L., \& Duerbeck, H. 1993, in Cataclysmic variables and related objects, NASA, 261

Harries, T. J., Hilditch, R. W., \& Howart, I. D. 2003, MNRAS, 339, 157

Jensen, E. L. N., Allen, P., Schwarz, G. J., \& Vanlandingham, K. M. 2001, IAUC, No. 7743

Kilmartin, P., \& Gilmore, A. C. 2002, IAUC, No. 7847

Liller, W. 2001, IAUC, No. 7738

Liller, W. 2002, IAUC, No. 7841

Massey, P., Lang, C. C., De Gioia Eastwood, K., \& Germany, C. 1995, ApJ, 438, 188
Mustel, E. R., \& Boyarchuk, A. A. 1970, Ap\&SS, 6, 183

Oestreicher, M. O., Gochermann, J., \& Schmidt-Kaler, T. 1995, A\&AS, 112, 495

Oestreicher, M. O., \& Schmidt-Kaler, T. 1996, A\&AS, 117, 303

Osterbrock, D. E. 1989, in Astrophysics of gaseous nebulae and active galactic nuclei (University Science Book)

Panagia, N. 1998, MmSAI, 69, 225

Payne-Gaposchkin, C. 1957, in Book review: The Galactic Novae, Science, 126, 1350

Pritchet, C. J., \& van den Bergh, S. 1987, ApJ, 318, 507

Schwering, P. B. W., \& Israel, F. P. 1991, A\&A, 246, 231

Shara, M. M., Zuerk, D. R., Williams, R. E., et al. 1997, AJ, 114, 258

Starrfield, S., Truran, J. W., Wiescher, M. C., \& Sparks, W. M. 1998 , MNRAS, 296, 502

Starrfield, S., Truran, J. W., Weischer, M. C., \& Sparks, W. M. 2000, ApJS, 127, 485

Subramaniam, A., \& Anupama, G. C. 2002, A\&A, 390, 449

Schwarz, G. J., Shore, S. N., Starrfield, S., et al. 2001, MNRAS, 320, 103

Sekiguchi, K., Caldwell, J. A. R., Stobie, R. S., \& Buckley, D. A. H. 1990, MNRAS, 245, 28

Shore, S. N., Schwarz, G., Bond, H. E., et al. 2003, AJ, 125, 1507

van den Bergh, S., \& Pritchet, C. J. 1986, PASP, 98, 110

van den Bergh, S., \& Younger, P. F. 1987, A\&AS, 70, 125

Vanlandingham, K. M., Starrfield, S., Shore, S. N., \& Sonneborn, G. 1999, MNRAS, 308, 577

Williams, R. E., Hamuy, M., Phillips, M. M., et al. 1991, ApJ, 376, 721

Williams, R. E. 1992, AJ, 104, 725

Williams, R. E. 1994, AJ, 426, 279

Williams, R. E., Phillips, M. M., \& Hamuy, M. 1994, ApJS, 90, 297

Williams, S. J., \& Shafter, A. W. 2004, ApJ, 612, 867 